of the symptoms preceding sudden and unexpected deaths at home when these are compared with their general rate of occurrence in the child population. Since the conclusions of the study are based on these findings some independent assessment of their reliability is desirable.

The conclusion of this paper is that the prevention of cot deaths is dependent firstly on the recognition by parents of the significance of certain non-specific symptoms and on their decision to involve the primary care services, and secondly on the efficacy of medical intervention at this stage. Consideration does not appear to have been given to the difficulties that will face parents who are asked to undertake such close surveillance of their children or the anxieties that this is likely to cause; no has any estimate been made of the presumably very large proportion of cases in which this will prove "unnecessary." Conversely, the implications of the increased work load for the primary care services has yet to be determined. Concerning intervention, the investigators recognise that the value of either hospital referral or drug therapy is at present doubtful, leaving close supervision and observation of the child at home as the only alternative. It has yet to be established what this can achieve.

By the nature of the condition, sudden and unexpected death in children is an exceedingly difficult area of study, especially in its community aspects, and many of the criticisms raised here are clearly recognised by the study group and are the subject of further investigation. But because of these unresolved difficulties it would seem premature to urge the "need to improve the recognition by both doctors and parents of non-specific symptoms as markers of severe illness in young children and their understanding of the necessity for rapid and appropriate action."

David GREAves ANDREW BODDY Social Paediatric and Obstetric
Research Unit, Glasgow

\section{Thyroid disease and pregnancy}

SIR,-We read with interest your leading article on this subject (7 October, p 977). You state that during pregnancy "free thyroid hormone concentrations are normal." The paper ${ }^{1}$ to which you refer states that "direct measurements of free serum $\mathrm{T} 4$ provide values similar to those seen in the non-pregnant state ... but there is some evidence that free T3 levels fall below normal and remain low until term."

We have recently completed a study of free T4 in the serum in the three trimesters of pregnancy using the Corning Immophase ${ }^{125} \mathrm{I}$ radioimmunoassay) method. The data provided with the kit currently show that the normal range and the range of values obtained during pregnancy are the same. Our results, which are in conflict both with those to which you refer and also with the Corning data, are summarised in the table. They show that in the second and third trimesters approximately onethird of the values obtained will be in the hypothyroid range.

Reports that serum free $\mathrm{T} 4$ concentrations were significantly lower than normal in pregnancy first appeared as long ago as $1958^{\circ}$ and $1962 .{ }^{3}$ Our results indicate that values not
Mean free T4 levels $( \pm S D)$ in pregnancy

\begin{tabular}{l|c|c}
\hline & $\begin{array}{c}\text { No of } \\
\text { patients }\end{array}$ & $\begin{array}{c}\text { Free T4 } \\
\text { (pmol/1) }\end{array}$ \\
\hline Controls & 22 & $16 \cdot 7.2 \cdot 4$ \\
1st trimester & 22 & $16 \cdot 73 \cdot 5$ \\
2nd trimester & 28 & $12 \cdot 54.5 *$ \\
3rd trimester & 19 & $9 \cdot 9 \pm 2 \cdot 7^{+}$
\end{tabular}

* Significant difference from control mean, $\mathrm{P}<0.001$ No significant difference from 2 nd trimester mean. Conversion: SI to traditional units-T $4: 1 \mathrm{pmol} / 1=0.08$
$\mathrm{ng} 100 \mathrm{ml}$.

significantly different from normal can be expected only in the first trimester. The combination of conflicting results and a novel technique for measuring free $\mathrm{T} 4$ could have a methodological basis. In order to clarify the situation our sera are now being assayed for free $\mathrm{T} 4$ and free $\mathrm{T} 3$ by the dialysis method," which is generally considered to be the reference method for these measurements.

If the dialysis measurements confirm our observation that the serum free $\mathrm{T} 4$ level falls during the second trimester and remains low until term then the widely taught concept that the free $\mathrm{T} 4$ concentration remains norma during pregnancy will have to be changed. Surely this will lead to some exciting new ideas about transport and action of thyroid hormones at cellular levels.

Margot Boss

O DJAHANBAKHCH

D KingSTONE

Departments of Obstetrics and
Gynaecology and Medical Physics,

Royal Free Hospital,

London NW3

Tunbridge, W M G, and Hall, R, Clinics in Obstetric and Gynaecology, 1975, 2, 38

Robbins, J, and Nelson, J H, fournal of Clinical Investigation, 1958, 37, 153 .

Sterling, K, and Hegedus, A, fournal of Clinical Investigation, 1962, 41, 1031 .

Ekins, R P, and Ellis, S M, Thyroid Research, 1976,

SIR,-I share the view of Dr R T Cooke (11 November, p 1370) that the published experience of postpartum hypothyroidism may be just the tip of the iceberg. Over the past 18 months in a general medical practice I have seen four patients ${ }^{1}$ with spontaneously remitting hypothyroidism presenting after delivery. I have also seen other women on thyroid replacement therapy for many years whose hypothyroidism was diagnosed after a delivery.

As the natural history of postpartum thyroid disorders is not known it is important that their remitting nature should be more widely appreciated.

\section{Whittington Hospital,}

B I HOFFBRAND

Hoffbrand, B I, and Webb, S C, Postgraduate Medical fournal. In press.

Towards positive diagnosis of the irritable bowel

SIR,-We were interested to read the article on this subject by Dr A P Manning and others (2 September, p 653), but had difficulty in interpreting the results.

Although it is stated that irritable bowe syndrome (IBS) is itself a diagnosis of exclusion, it seems curious that 27 patients in the original sample of 109 were excluded because no definite diagnosis had been reached. Did the symptoms in this group of patients, and those in the group of 14 excluded because of (perhaps coincidental) diverticular disease, differ from the symptoms of the group of 32 with IBS ?

Is it not possible that patients with upperbowel disease present with a different range of symptoms from those in patients with conditions affecting mainly the lower bowel? Twenty of the 33 patients with an organic diagnosis had conditions affecting the upper gastrointestinal tract and the differences between the symptomatology of this group and that of the group with IBS could be entirely ascribed to this distinction. Perhaps the article could be retitled "Towards positive diagnosis of upper bowel disease"?

Sarah Pearson

PETER OPENSHAW Medical students

Guy's Hospital,

London SE1

** We sent a copy of this letter to Dr Manning and his colleagues, whose reply is printed below.-ED, $B M \mathcal{H}$.

SIR,-The 27 patients who were omitted from our study were a heterogeneous group. Some had functional dyspepsia, some psychoneurosis, and in some no definite conclusion was reached in the clinic-for example, because the symptoms resolved spontaneously or the patient defaulted from follow-up. Statistically they were different from the IBS group, but it is hard to draw any conclusions from this. There were only 14 patients in the diverticular disease group-not enough for meaningful statistical comparisons with the IBS and organic groups.

We agree that the IBS patients could be considered as a group of people with mainly colonic and rectal dysfunction whereas the organic patients were heterogeneous but predominantly with upper-gut problems. This does not alter the validity of our findings. In clinical practice it is often not at all obvious if a patient's abdominal pain is coming from the foregut, midgut, or hindgut (colon pain is often felt at or above the umbilicus). To identify the hindgut as the source of pain should not only save unnecessary cholecystograms, barium meals, endoscopies, and even intravenous pyelograms but also allow the doctor to explain the probable nature of the disorder at the first interview. It may, of course, be necessary to exclude an organic lesion in the hindgut, but there are usually clues to this such as blood in the stool, weight loss, or sigmoidoscopic abnormalities.

In our experience patients with IBS often suffer from gastric symptoms which improve when the IBS is treated, and there is experimental evidence that upper-gut dysfunction is common in IBS. These cases of IBS are missed unless the doctor asks colon-directed questions.

Our statement that the IBS is a diagnosis of exclusion was a reference to conventional thinking. We challenge this attitude and suggest that doctors should have more confidence in their history-taking. By spending more time on the history they can not only avoid negative investigations and referrals but also increase their job satisfaction.

A P MANNING

W G THOMPSON $\mathrm{K}$ W HEATON

University Department of Medicine, Bristol Royal Infirmary, 